\title{
Tc-99m pyrophosphate imaging for transthyretin cardiac amyloidosis: Importance of estimation of cancer risk with the modality
}

\author{
Shivda Pandey, MBBS, ${ }^{a}$ and Omar K. Siddiqi, $M^{a, b}$ \\ a Section of Cardiovascular Medicine, Department of Medicine, Boston University School of \\ Medicine, Boston Medical Center, Boston, MA \\ b Amyloidosis Center, Boston University School of Medicine, Boston Medical Center, Boston, \\ MA
}

Received Jun 25, 2018; accepted Jun 25, 2018

doi: $10.1007 / \mathrm{s} 12350-018-1362-0$

\section{See related article, pp. 215-224}

Transthyretin cardiac amyloidosis (ATTR-CA) is a progressive restrictive cardiomyopathy, marked by extracellular deposition of misfolded genetically abnormal (mutant ATTR or ATTRm) or genetically normal (wild-type ATTR or ATTRwt) transthyretin protein (previously prealbumin) manufactured by the liver. Previously thought to be a rare disease, ATTRwt cardiac amyloidosis may account, variably, for between $5 \%$ and $18 \%$ of patients over the age of 65 years with heart failure with preserved ejection fraction $(\mathrm{HFpEF}){ }^{1,2}$ Furthermore, specific pathogenic mutations in the transthyretin gene are particularly prevalent in certain populations, with the valine to isoleucine substitution at position 122 (Val122Ile) affecting 3.2\% of African Americans ${ }^{3}$ with a prevalence of $5 \%$ in African American patients over the age of 60 with clinical heart failure ${ }^{4}$. Additionally, recent evidence points to relatively high prevalence of ATTRwt cardiac amyloidosis in patients with aortic stenosis (AS). Histopathologic evaluation of cardiac tissue obtained from post-transcatheter aortic valve replacement (TAVR) patients either at the time of autopsy or after valve

Reprint requests: Shivda Pandey, MBBS, Section of Cardiovascular Medicine, Department of Medicine, Boston University School of Medicine, Boston Medical Center, 88 East Newton Street, Boston, MA; Shivda.Pandey@bmc.org

J Nucl Cardiol 2020;27:225-7.

$1071-3581 / \$ 34.00$

Copyright (C) 2018 American Society of Nuclear Cardiology. explantation revealed ATTR cardiac amyloidosis in onethird of the cases ${ }^{5}$. Another study demonstrated that occult wild-type ATTR-CA had $6 \%$ prevalence in patients with AS aged $>65$ years referred for surgical aortic valve replacement and was associated with poor outcome ${ }^{6}$. In patients with severe calcific AS undergoing TAVR, ATTR-CA was present in $16 \%$ of cases and was associated with low-flow low-gradient AS (LF-LG AS) with mildly reduced $\mathrm{LVEF}^{7}$. These studies used endomyocardial biopsy and scintigraphy with bone-avid tracers (99mTc-3,3-diphosphono-1,2-propanodicarboxylic acid or of $99 \mathrm{mTc}$-pyrophosphate) for diagnosis of ATTR-CA.

Bokhari et al. have described the diagnostic accuracy of 99mTc-pyrophosphate (99mTc-PYP) scintigraphy in identifying patients with ATTR and distinguishing ATTR from light-chain amyloidosis $(\mathrm{AL})^{8}$. Therefore, $99 \mathrm{mTc}-\mathrm{PYP}$ is now being effectively used to non-invasively diagnose ATTR-CA. Timely identification of ATTR-CA provides valuable prognostic insight and facilitates selection of appropriate treatment. This is particularly important with the recent development of targeted therapies that involve TTR stabilization, TTR suppression, and TTR disruption ${ }^{9}$. Data suggest a decrease in the combined primary outcome of mortality and heart transplantation in patients treated with the TTR stabilizers diflunisal and tafamidis ${ }^{10}$. Furthermore, certain important considerations exist with medical management of heart failure in amyloid cardiomyopathy-for example caution with use of beta-blockers, which are often poorly tolerated in this group of patients ${ }^{9}$.

Given the prevalence of ATTR-CA in patients with HFpEF, and aortic stenosis, as well as in African Americans, and the associated prognostic and 
therapeutic implications of this diagnosis, 99mTc-PYP scintigraphy has the potential to evolve into a screening modality for this high-risk group of patients. Use of 99mTc-PYP scintigraphy for screening purposes will lead to widespread application and therefore, it is extremely essential to evaluate the risk of radiation exposure associated with the test.

In the current issue of the journal, Einstein et al. ${ }^{14}$ performed an analysis to estimate cancer-risk associated with radiation exposure from 99mTc-PYP scintigraphy for cardiac amyloidosis work-up. Organ-specific absorbed radiation doses per unit of administered activity were determined using dose coefficients from Medical Internal Radiation Dose (MIRD) Committee of the Society of Nuclear Medicine and Molecular Imaging using a model developed specifically for $99 \mathrm{mTc}-\mathrm{PYP}^{11}$. The dose coefficients obtained through MIRD were also compared to those listed by International Commission on Radiological Protection (ICRP) for 99mTc-PYP-labeled phosphates and phosphonates ${ }^{12}$. RadRAT (Radiation risk assessment tool) estimates radiation-attributable cancer risks using data from the Life Span Study cohort ${ }^{13}$. The authors used RadRAT to obtain the following risk estimates: excess future risk from $99 \mathrm{mTc}-$ PYP, baseline future risk, and the percentage of risk from $99 \mathrm{mTc}-\mathrm{PYP}$ (excess divided by excess plus baseline). Although the study estimated organ doses for $99 \mathrm{mTc}-\mathrm{PYP}$ protocol with $10 \mathrm{mCi}$ of administered activity, the authors added that the estimated risks would double by increasing the dose to $20 \mathrm{mCi}$, since some labs use the higher dose. Surveillance Epidemiology and End Results (SEER) data were used to obtain baseline cancer incidence rates.

Findings demonstrated that the highest excess 99mTc-PYP-related risk of cancer was that of urinary bladder cancer, with a risk ( $90 \%$ uncertainty range) of $9.45(2.48,22.6)$ per 100,000 men scanned and 8.98 $(2.61,20.5)$ per 100,000 women scanned at age 40 . The risks decreased with increase in age. Baseline future risk of bladder cancer in men at 40 years of age was 3840 per 100,000 scans with $90 \%$ uncertainty range being 3770 to 3920 and therefore excess risk from $99 \mathrm{mTc}-$ PYP in that group was $0.25 \%$. Similarly, excess risk from $99 \mathrm{mTc}-\mathrm{PYP}$ in 40 year old women was estimated as $0.74 \%$. To put these findings into perspective, there is less than $1 \%$ chance that urinary bladder cancer in a 40 year old man or woman is attributable to radiation exposure from a $99 \mathrm{mTc}-\mathrm{PYP}$ scan.

The authors presented other risk estimates succinctly in the tables.

Several caveats exist with interpretation of the above findings. Radiation dosimetry estimates for radiopharmaceuticals are generally based on limited pharmacokinetic data from a small group of patients and use compartment models assuming a median weight for the patients ${ }^{11,14}$. As the authors pointed out, MIRD dosimetry estimates were obtained from data from 15 patients and there is no information regarding specific patient demographics. While average dosimetry and cancer risks are estimated for a population, the risk estimates may not be applicable for a particular patient with a particular imaging-related exposure ${ }^{14}$. The main aim of this study is estimation of cancer-risk associated with radiation exposure from diagnostic imaging which uses the linear no threshold hypothesis (LNT). There is considerable uncertainty in the validity and application of the LNT hypothesis to low-level radiation exposure that typically occurs with imaging studies since data on cancer risk estimates from radiation exposure is mostly derived from atomic bomb survivors with high-dose radiation exposure ${ }^{14}$. Lifetime-risk estimates take into account life expectancy of the study population, which may not be comparable to the general population as mentioned by the authors. At-risk individuals undergoing $99 \mathrm{mTc}-\mathrm{PYP}$ scintigraphy may have lower than average life expectancy, in which case attributable cancer risk will be overestimated and risk-benefit ratio would favor $99 \mathrm{mTc}-\mathrm{PYP}$ scan for timely diagnosis and management of these patients.

In summary, ATTR-CA is likely an under-diagnosed disease entity and has been associated with HFpEF and aortic stenosis. ATTR-CA due to the Val122Ile mutation may be particularly prevalent in African American patients. Presence of ATTR-CA adversely impacts prognosis and accurate diagnosis is essential for appropriate risk stratification and management. 99mTc-PYP scintigraphy has high diagnostic accuracy for detection of ATTR-CA and has the potential to evolve into a screening tool in the at-risk population. Einstein et al. ${ }^{14}$ conducted a comprehensive analysis to estimate attributable cancer risks with 99mTc-PYP scintigraphy used to diagnose TTR-CA. Although the study findings are based on numerous assumptions, they suggest very low cancer risks associated with 99mTc-PYP scintigraphy.

\section{References}

1. Mohammed SF, Mirzoyev SA, Edwards WD, Dogan A, Grogan DR, Dunlay SM, et al Left ventricular amyloid deposition in patients with heart failure and preserved ejection fraction. J Am Coll Cardiol HF. 2014;2:113-22.

2. González-López E, Gallego-Delgado M, Guzzo-Merello G, de Haro-Del Moral FJ, Cobo-Marcos M, Robles C, et al Wild-type transthyretin amyloidosis as a cause of heart failure with preserved ejection fraction. Eur Heart J. 2015;36:2585-94.

3. Buxbaum J, Alexander A, Koziol J, Tagoe C, Fox E, Kitzman D. Significance of the amyloidogenic transthyretin Val 122 ile allele 
in african Americans in the arteriosclerosis risk in communities (ARIC) and cardiovascular health (CHS) studies. Am Heart J. 2010;159(5):864-70.

4. Arvanitis M, Chan GG, Jacobson DR, Berk JL, Connors LH, Ruberg FL. Prevalence of mutant ATTR cardiac amyloidosis in elderly African Americans with heart failure. Amyloid. 2017;24:253-5.

5. Nietlispach F, Webb JG, Ye J, Cheung A, Lichtenstein SV, Carere RG, et al Pathology of transcatheter valve therapy. J Am Coll Cardiol Intv. 2012;5:582-90.

6. Treibel TA, Fontana M, Gilbertson JA, Castelletti S, White SK, Scully PR, et al Occult transthyretin cardiac amyloid in severe calcific aortic stenosis: Prevalence and prognosis in patients undergoing surgical aortic valve replacement. Circ Cardiovasc Imaging. 2016;9:e005066.

7. Castaño A, Narotsky DL, Hamid N, Khalique OK, Morgenstern R, DeLuca A, et al Unveiling transthyretin cardiac amyloidosis and its predictors among elderly patients with severe aortic stenosis undergoing transcatheter aortic valve replacement. Eur Heart J. 2017;38:2879-87.

8. Bokhari S, Castaño A, Pozniakoff T, Deslisle S, Latif F, Maurer MS. 99mTc-pyrophosphate scintigraphy for differentiating lightchain cardiac amyloidosis from the transthyretin-related familial and senile cardiac amyloidoses. Circ Cardiovasc Imaging. 2013;6:195-201.

9. Siddiqi OK, Ruberg FL. Cardiac amyloidosis: An update on pathophysiology, diagnosis, and treatment. Trends Cardiovasc Med. 2018;28:10-21.

10. Rosenblum H, Castano A, Alvarez J, Goldsmith J, Helmke S, Maurer MS. TTR (Transthyretin) Stabilizers Are Associated With Improved Survival in Patients With TTR Cardiac Amyloidosis. Circ Heart Fail. 2018;11(4):e004769.

11. Weber DA, Makler PT Jr, Watson EE, Coffey JL, Thomas SR, London J. Imaging Agents. J Nucl Med. 1989;30:1117-22.

12. Mattsson S, Johansson L, Leide Svegborn S, Liniecki J, Noßke D, Riklund $\mathrm{KA}$, et al Radiation dose to patients from radiopharmaceuticals: A compendium of current information related to frequently used substances. Ann ICRP. 2015;44:7-321.

13. Berrington de Gonzalez A, Iulian Apostoaei A, Veiga LH, Rajaraman P, Thomas BA, Owen Hoffman F, Gilbert E, et al RadRAT: a radiation risk assessment tool for lifetime cancer risk projection. J Radiol Prot. 2012;32:205-22.

14. Ep Ficaro, Zanzonico P, Stabin MG, Raff GL, Thompson RC, Einstein AJ, et al Variability in radiation dose estimates from nuclear and computed tomography diagnostic imaging. JNC. 2009. https://doi.org/10.1007/s12350-008-9026-0. 